\title{
ON PERMUTATIONAL PRODUCTS OF GROUPS
}

PART 2 - AMALGAMATED PRODUCTS

\author{
R. J. GREGORAC
}

(Received 16 October 1968; revised 14 June 1969)

To Bernhard Hermann Neumann on his 60th birthday

Communicated by G. E. Wall

\section{Introduction}

The standard methods of constructing generalized free products of groups (with a single amalgamated subgroup) and permutational products of groups are to consider groups of permutations on sets. Although there is an apparent similarity between these two constructions, the exact nature of the relationship is not clear. The following addendum to [4] grew out of an attempt to determine this relationship. By noting that the original construction of permutational products (B. H. Neumann [7]) deals with a group of permutations on a group (although the group structure has been previously ignored; see [7], [8]) we here give an extension of the original permutational product-construction which yields both the generalized free product and the permutational products as groups of permutations on groups. A generalized free product is represented as a group of permutations on the ordinary free product of the constituents of the underlying group amalgam and a permutational product is a group of permutations on the direct product of the constituents of the amalgam.

It is also shown that this construction can be extended to other groups $G$ containing the constituents of the amalgam provided certain conditions hold; to differentiate the general case from ordinary permutational products we call the groups of permutations so obtained amalgamated products.

As in [4] an epimorphism can be constructed between suitable amalgamated products and the wreath product embeddings of permutational products given in [4] can then be extended to certain amalgamated products.

Finally, this construction also yields a class of related generalized regular products (Theorem 4.6), which, so far as we know, is the only such class known, besides ordinary permutational products (Allenby [2]) and some classes which have been shown to exist by Wiegold [12].

\section{Preliminaries}

If $x$ and $y$ are elements of a group $G$, write $y^{-1} x y=x^{y}$ and $x^{-1} y^{-1} x y=$ $[x, y]$. Note that all mappings act on the right. If $X_{i}(i \in I)$ are subgroups of $G$, 
then $\left[X_{i}\right]$ denotes the subgroup of $G$ generated by $\left\{\left[x_{i}, x_{j}\right] \mid x_{i} \in X_{i}, x_{j} \in X_{j}, i \neq j\right.$, $i, j \in I\}$ and $X_{i}^{G}$ the normal closure $X_{i}\left[X_{i}, G\right]$ of $X_{i}$ in $G$. We shall say that a group $G$ generated by subgroups $X_{i}(i \in I)$ is a regular product of the $X_{i}$, if $G \cong F / N$, where $F=\Pi *\left\{X_{i} \mid i \in I\right\}$ is the (ordinary) free product of the $X_{i}$ and $N \subseteq\left[X_{i}\right]^{F}$ (Golovin [3]). Assume now that the index set $I$ is ordered.

THEOREM 2.1 [3]. If a group $G$ is generated by subgroups $X_{i}(i \in I)$, then $G$ is a regular product of the $X_{i}$ if and only if every element $g$ if $G$ has a unique regular representation

where $x_{k} \in X_{i_{k}}, u \in\left[X_{i}\right]^{G}$ and

$$
g=x_{1} x_{2} \cdots x_{n} u
$$

$$
i_{1}<i_{2}<\cdots<i_{n} .
$$

If $V$ is a set of words, let $V(G)$ denote the $V$-verbal subgroup of $G$, i.e., the subgroup of $G$ generated by all values of the words of $V$ in $G$.

Definition 2.2 (Moran [5]). Let $V$ be a set of words. The V-verbal product $\Pi_{V}^{*}\left\{X_{i} \mid i \in I\right\}$ of groups $X_{i}$ is $F / V(F) \cap\left[X_{i}\right]^{F}$, where $F=\Pi^{*}\left\{X_{i} \mid i \in I\right\}$.

THEOREM 2.3 [5]. If $G=\Pi_{V}^{*}\left\{X_{i} \mid i \in I\right\}$ and $I=I_{1} \cup I_{2}$, where $I_{1} \cap I_{2}$ is empty, then the subgroups generated by the $X_{i}\left(i \in I_{1}\right)$ and $X_{j}\left(j \in I_{2}\right)$ are, respectively, $G_{1}=\Pi_{V}^{*}\left\{X_{i} \mid i \in I_{1}\right\}$ and $G_{2}=\Pi_{V}^{*}\left\{X_{j} \mid j \in I_{2}\right\}$, and $G=G_{1} *_{V} G_{2}$.

THEOREM 2.4 [6]. If $X_{i}(i \in I)$ are groups and $\phi_{i}$ is a homomorphism of the group $X_{i}$ for each $i \in I$, then there exists a homomorphic mapping $\phi$ of $\Pi_{V}^{*}\left\{X_{i} \mid i \in I\right\}$ onto $\Pi_{V}^{*}\left\{X_{i} \phi_{i} \mid i \in I\right\}$ whose restriction to the group $X_{i}$ is $\phi_{i}$ for every $i \in I$.

Suppose for each $i \in I, A_{i}$ is a group containing a subgroup $H_{i}$ which is isomorphic to a fixed group $H$, say $\psi_{i}: H_{i} \cong H$. Let $\psi_{i j}=\psi_{i} \psi_{j}^{-1}$. We change the notation of [4] and define an amalgam of the $A_{i}$ amalgamating the $H_{i}$ according to the $\psi_{i j}$ to be the system $\left(A_{i}, H_{i}, \psi_{i j} ; i, j \in I\right)$. We denote this amalgam by $\mathscr{A}=\operatorname{Am}\left(A_{i}, H_{i}, \psi_{i j} ; i, j \in I\right)$ and ordinarily think of the $H_{i}$ as being identified by the $\psi_{i j}$ so the amalgam becomes the union of the $A_{i}$ intersecting in $H$ (or $H_{1}$ ). The $A_{i}$ are called the constituents of the amalgam and $H$ is the amalgamated subgroup.

A group $G$ embeds the amalgam $\mathscr{A}$ if there exist isomorphisms $\phi_{i}: A_{i} \rightarrow$ $A_{i}^{\prime} \subseteq G$ such that (i) $A_{i}^{\prime} \cap A_{j}^{\prime}=H^{\prime} \subseteq G$, (ii) if $h \in H$, then $h \psi_{i}^{-1} \phi_{i}=h \psi_{j}^{-1} \phi_{j}$ and (iii) if $h^{\prime} \in H^{\prime}$, then $h^{\prime} \phi_{i}^{-1} \in H_{i}$ and $h^{\prime} \phi_{i}^{-1} \psi_{i}=h^{\prime} \phi_{j}^{-1} \psi_{j}(i, j \in I)$.

The group $G$ will be said to be generated by the amalgam $\mathscr{A}$, if $G$ embeds $\mathscr{A}$ and is generated by the embedded copy of $\mathscr{A}$.

If $G$ is the generalized free product on $\mathscr{A}$ (this can be defined as the group constructed in the following Example (3.6) (2)), then $K$ is called a generalized regular product on $\mathscr{A}$, if $K$ embeds $\mathscr{A}$ and $K \cong G / N$, where $N$ is a normal subgroup of $G$ contained in $\left[A_{i}\right]^{G}$ (Wiegold [12]). 
DEFINITION 2.5 [11]. If $V$ is a set of words, the group $G$ is a generalized $V$-verbal product of its subgroups $G_{\alpha}(\alpha \in M)$ with amalgamations $G_{\alpha} \cap G_{\beta}=H_{\alpha \beta}(\alpha \neq \beta)$, if

(i) $G$ is generated by the $G_{\alpha}(\alpha \in M)$ and

(ii) $V(G) \cap\left[G_{\alpha}\right]^{G}=\{1\}$.

THEOREM 2.6 [12]. If the free generalized $V$-product of $A$ and $B$ amalgamating $H$ and $H \phi$ according to $\phi$ exists it is $G_{0} / N$, where

(i) $G_{0}$ is the $V$-verbal product of $A$ and $B$ and

(ii) $N$ is the normal closure in $G_{0}$ of the set of all elements of the form $h^{-1}(h \phi)$, where $h$ ranges over $H$.

Lemma 2.7. ([11], Lemma 7.9). Let $G$ be any group and $g, d \in G$ such that $\left[d^{2}, g\right]=1$. Then for each $r \geqq 0\left[g^{2^{r}}, d\right]$ is, in the $(r+1)$-st term of the lower central series of $G, G_{(r+1)}$.

\section{The construction}

For simplicity we deal with only two groups here; an extension to an arbitrary number of groups will be indicated later.

Let $A m\left(A_{1}, A_{2} ; H_{1}, H_{2} ; \psi\right), \psi_{12}=\psi$, be a given group amalgam. Suppose $G$ is any group containing isomorphic copies $A_{i}^{*}$ of $A_{i}(i=1,2)$, where $\phi_{i}: A_{i} \cong A_{i}^{*}$ $(i=1,2)$, such that

(i) $A_{1}^{*} \cap A_{2}^{*}=H_{1}^{*} \cap H_{2}^{*}$, where $H_{i}^{*}=H_{i} \phi_{i}(i=1,2)$, and

(ii) the isomorphism $\psi^{*}=\left.\left.\phi_{1}^{-1}\right|_{H_{1}^{*}} \psi \phi_{2}\right|_{H_{2}}$ from $H_{1}^{*}$ onto $H_{2}^{*}$ acts as the identity when restricted to $H_{1}^{*} \cap H_{2}^{*}$.

Let $H$ be the subgroup of $G$ generated by $H_{1}^{*}$ and $H_{2}^{*}$ and suppose $H \cap A_{2}^{*}=$ $H_{2}^{*}$. Let $G=\cup z H, z \in Z$, be a coset decomposition of $G$ relative to $H$. Assume further that there is an automorphism $\tau$ (called a switching map) of $H$ such that $\tau \mid H_{1}^{*}=\psi^{*}$ and $\tau \mid H_{2}^{*}=\psi^{*-1}$. Note that $\tau^{2}=1$. Next let $\sigma$ be any permutation on $Z$ of order two which fixes the coset representative of $H$ and such that for all $z \in Z$, if $A_{2}^{*} \backslash H_{2}^{*}$ meets $z H$, then $A_{1}^{*} \cap(z \sigma) H$ is empty. (E.g., see Example 3.6 (1) which follows.) Define a function $\pi$ on $G$ by

$$
(z h) \pi=(z \sigma)(h \tau), \quad \text { for } z \in Z, h \in H .
$$

Clearly $\pi^{2}=1$, so $\pi=\pi^{-1}$ and $\pi \in \mathscr{S}(G)$, the group of all permutations on $G$. Finally, assume $(1 \pi) A_{2}^{*} \subseteq A_{2}^{*} H$.

Let $\rho: G \rightarrow \mathscr{S}(G)$ be the right regular representation of $G$. We shall now prove that the amalgam $\mathscr{A}=A_{1}^{*} \rho \cup \pi^{-1}\left(A_{2}^{*} \rho\right) \pi$ is a copy of $\operatorname{Am}\left(A_{1}, A_{2} ; H_{1}, H_{2}\right.$; $\psi$ ) embedded in $\mathscr{S}(G)$. The subgroup of $\mathscr{S}(G)$ generated by the amalgam $\mathscr{A}$ will be the required product. 
Clearly $A_{1}^{*} \rho \cong A_{1}$ and $\pi^{-1}\left(A_{2}^{*} \rho\right) \pi \cong A_{2}^{*} \rho \cong A_{2}$. We first show that $H_{1}^{*} \rho=$ $\pi\left(H_{2}^{*} \rho\right) \pi\left(\right.$ recall $\left.\pi=\pi^{-1}\right)$.

Let $h_{1} \in H_{1}^{*}$ and denote the image of $h_{1}$ under $\rho$ by $\rho_{h_{1}}$. Then

$$
\rho_{h_{1}}=\pi \rho_{u} \pi, \quad \text { where } u=h_{1} \psi^{*},
$$

for if $z h \in G, z \in Z, h \in H$, then

$$
\begin{array}{rlr}
(z h) \pi \rho_{u} \pi & =(z \sigma h \tau) \rho_{u} \pi & \\
& =\left(z \sigma h \tau h_{1} \psi^{*}\right) \pi & \\
& =\left(z \sigma\left(h h_{1}\right) \tau\right) \pi & \left(\psi^{*}=\tau \mid H_{1}^{*}\right) \\
& =z\left(h h_{1}\right) & \left(\sigma^{2}=\tau^{2}=1\right) \\
& =(z h) \rho_{h_{1}} . &
\end{array}
$$

Now let

$$
\rho_{a_{1}}=\pi \rho_{a_{2}} \pi \in A_{1}^{*} \rho \cap \pi\left(A_{2}^{*} \rho\right) \pi .
$$

where $a_{1} \in A_{1}^{*}$ and $a_{2} \in A_{2}^{*}$. Then

$$
1 \rho_{a_{1}}=a_{1} \in A_{1}^{*} .
$$

Let $1=z h$, where $z$ represents $H$ and $h \in H$. Note $1 \pi=(z \sigma)(h \tau)=z(h \tau) \in H$ and

$$
\begin{aligned}
& \text { (1) } \pi \rho_{a_{2}} \pi=\left(1 \pi a_{2}\right) \pi \\
& =\left(a_{2}^{\prime} h^{\prime}\right) \pi, a_{2}^{\prime} \in A_{2}^{*}, h^{\prime} \in H \quad\left((1 \pi) A_{2}^{*} \subseteq A_{2}^{*} H\right) \\
& =\left(z^{\prime} \sigma\right)\left(h^{\prime \prime} \tau\right) \text {, }
\end{aligned}
$$

where $a_{2}^{\prime} h^{\prime}=z^{\prime} h^{\prime \prime}, z^{\prime} \in Z, h^{\prime \prime} \in H$. By (3.3) and (3.4),

$$
\left(z^{\prime} \sigma\right)\left(h^{\prime \prime} \tau\right)=a_{1} \in A_{1}^{*},
$$

so $\left(z^{\prime} \sigma\right) H$ meets $A_{1}^{*}$; hence no element of $A_{2}^{*} \backslash H_{2}^{*}$ can be written as $z^{\prime} h^{*}, h^{*} \in H$, that is, $a_{2}^{\prime} \in H_{2}^{*}$. Since $1 \pi \in H, a_{2}=(1 \pi)^{-1} a_{2}^{\prime} h^{\prime}$ must also be in $H \cap A_{2}^{*}=H_{2}^{*}$. If $a_{2}=a_{1}^{*} \psi^{*}$, for some $a_{1}^{*} \in H_{1}^{*}$, then $1 \pi a_{2}=z(h \tau)\left(a_{1}^{*} \psi^{*}\right)=z\left(h a_{1}^{*}\right) \tau$ and $\left(1 \pi a_{2}\right) \pi$ $=(z h) a_{1}^{*}$. But $a_{1}^{*}=a_{1}$ by (3.5), so $a_{1} \in H_{1}^{*}$ and as in (3.2) $a_{2}=a_{1} \psi^{*}$. Hence $H_{1}^{*} \rho=A_{1}^{*} \rho \cap \pi\left(A_{2}^{*} \rho\right) \pi$, as required.

The group $P(G, Z, \sigma)$ generated by $\mathscr{A}$ in $\mathscr{S}(G)$ will be called a $(G, Z, \sigma)$ amalgamated product on $\mathscr{A}$, or more briefly, a $(G, Z, \sigma)$-product on $\mathscr{A}$.

(3.6) EXAMPLES. Throughout the following examples we consider the given amalgam $\mathscr{A}=\operatorname{Am}\left(A_{1}, A_{2} ; H_{1}, H_{2} ; \psi\right)$.

Let $G=A_{1} * A_{2} / N$ be any ordinary regular product of $A_{1}$ and $A_{2}$ (we assume here that $\left.A_{1}^{*}=A_{i}(i=1,2)\right)$. Then (i) and (ii) hold trivially because $H_{1} \cap H_{2}=1$. It follows from the unique normal form for elements of $G$ that $H \cap A_{2}=H_{2}$, for, if $a_{2} \in A_{2} \cap H$ and $a_{2}=h_{1} h_{2} c, h_{1} \in H_{1}, h_{2} \in H_{2}, c \in\left[A_{1}, A_{2}\right]$, then 
$1=h_{1} a_{2}^{-1}\left[a_{2}^{-1}, h_{1}\right] h_{2} c=h_{1}\left(a_{2}^{-1} h_{2}\right) c^{\prime}, c^{\prime} \in\left[A_{1}, A_{2}\right], \quad$ so $a_{2}^{-1} h_{2}=1$,

that is, $a_{2} \in H_{2}$.

Choose a transversal $Z$ of $H$. There is always at least one permutation $\sigma$ satisfying the hypotheses of the construction, namely, the identity $i$ on $Z$. For, if $z H$ meets $A_{2} \backslash H_{2}$, then $z h^{*}=a_{2} \in A_{2} \backslash H_{2}$, so $z=h_{1}^{*} a_{2}^{*} c, h_{1}^{*} \in H_{1}, a_{2}^{*} \in A_{2} \backslash H_{2}$, $c \in\left[A_{1}, A_{2}\right]$. If $z h=a_{1} \in A_{1}$, then $h_{1}^{*} a_{2}^{*} c h=a_{1}$, that is, $h_{1}^{* *} a_{2}^{* *} c^{*}=a_{1}, h_{1}^{* *} \in H_{1}$, $a_{2}^{* *} \in A_{2} \backslash H_{2}, c^{*} \in\left[A_{1}, A_{2}\right]$, which is impossible by the uniqueness of the normal form for elements. Thus $z H \cap A_{1}$ is empty. Finally, if $\tau$ exists and $1 \in Z$, then $1 \pi=1$, so the condition $(1 \pi) A_{2} \subseteq A_{2} H$ can also always be satisfied here. The main problem is, of course, the existence of $\tau$. We now consider some important cases where $\tau$ can be shown to exist.

(1) Permutational Products. Let $G=A_{1} \times A_{2}$; then $H=H_{1} \times H_{2}$ and the switching map $\tau$ exists, since it merely sends $\left(h_{1}, h_{2}\right) \in H$ to $\left(h_{2} \psi^{-1}, h_{1} \psi\right)$ which evidently defines an automorphism on $H$. Let $S_{i}$ be any transversal of $H_{i}$ in $A_{i}$ $(i=1,2)$, choose $S_{1} \times S_{2}$ as the transversal $Z$ of $H$ in $G$ and let $\sigma$ be the identity on $Z$. Note $H A_{2}=A_{2} H$, so $(1 \pi) A_{2} \subseteq A_{2} H$. The group $P=P\left(A_{1} \times A_{2}, S_{1} \times S_{2}, i\right)$ is a permutational product on $\mathscr{A}$ as originally described by B. H. Neumann [7] in 1954. (The term 'permutational product' was given by B. H. Neumann in 1960 ([8]) to a certain permutation group on $S_{1} \times S_{2} \times H_{1}$ which is isomorphic to $P$ above.)

(2) Generalized Free Products. Let $G=A_{1} * A_{2}$, the ordinary free product on $A_{1}$ and $A_{2}$; then $H=H_{1} * H_{2}$. By the fundamental property of free products the isomorphisms $\psi: H_{1} \rightarrow H_{2} \subseteq H$ and $\psi^{-1}: H_{2} \rightarrow H_{1} \subseteq H$ can be extended to a homomorphism $\tau$ from $H$ onto $H$. Since $\tau^{2}=1, \tau$ is an automorphism on $H$, i.e., the switching map exists. Let $Z$ be any transversal of $H$ in $G$ containing 1 and let $\sigma$ be $i$. If $\chi \in P=P\left(A_{1} * A_{2}, Z, i\right)$, then without loss of generality

$$
\chi=\rho_{a_{1}} \rho_{a_{2}}^{\pi} \cdots \rho_{a_{n-1}}^{\pi} \rho_{a_{n}}
$$

where, if $\chi \notin H_{1} \rho$, then it can be assumed that $a_{i} \in A_{1} \backslash H_{1}, i=1,3, \cdots, n$ and $a_{i} \in A_{2} \backslash H_{2}, i=2,4, \cdots, n-1$. If $n \geqq 1$, and $\chi \notin H_{1} \rho, \chi$ is said to have length $n$; otherwise $\chi$ has length zero.

In order to show $P$ is the generalized free product on $\mathscr{A}$ it suffices to show that $\chi$ is non-trivial whenever the length $n \geqq 1$. The action of $\rho_{a_{1}}$ on $1 \in A_{1}: A_{2}$ is $1 \rho_{a_{1}}=a_{1}=\left(a_{1} h_{1}^{-1}\right) h_{1}$, where $a_{1} h_{1}^{-1} \in Z, h_{1} \in H$, so

$$
\text { (1) } \begin{aligned}
\rho_{a_{1}} \pi \rho_{a_{2}} & =a_{1} h_{1}^{-1} h_{1}^{\tau} a_{2} \\
& =\left(a_{1} h_{1}^{-1} h_{1}^{\tau} a_{2} h_{2}^{-1}\right) h_{2}
\end{aligned}
$$

where $a_{1} h_{1}^{-1} h_{1}^{\tau} a_{2} h_{2}^{-1} \in Z, h_{2} \in H$ and (1) $\rho_{a_{1}} \rho_{a_{2}}^{\pi}=a_{1} h_{1}^{-1} h_{1}^{\tau} a_{2} h_{2}^{-1} h_{2}^{\tau}$.

Continuing this process,

$$
1 \chi=a_{1} h_{1}^{-1} h_{1}^{\tau} a_{2} h_{2}^{-1} h_{2}^{\tau} \cdots a_{n} \in A_{1} * A_{2} .
$$


Assume all pairs $h_{j}^{-1} h_{j}^{\tau}$ are written in normal form as elements of $A_{1} * A_{2}$. Suppose $a_{j} \in A_{1}$. Since $a_{j} \notin H, h_{1}^{*} a_{j} h_{2}^{*} \notin H$ for any $h_{1}^{*}, h_{2}^{*} \in H$; in particular $h_{1}^{*} a_{j} h_{2}^{*} \notin H_{1}$ for any $h_{1}^{*}, h_{2}^{*} \in H_{1}$. Therefore only contractions, but no cancellation, can occur between the $h_{j}^{-1} h_{j}^{\tau}$ and $a_{j}$ when reducing $1 \chi$ to normal form. Thus, $1 \chi \neq 1$, which was to be shown.

(3) A retraction $\phi$ of a group $G$ is an idempotent endomorphism of $G$, i.e. $\phi^{2}=\phi: G \rightarrow G$. If $H=G \phi$, then $H$ is called a retract of $G$.

Lemma 3.8 (Šmel'kin [10]). Let $G=A_{1} *_{V} A_{2}$ be a V-verbal product of $A_{1}$ and $A_{2}$. Suppose $\phi_{i}$ is a retraction of $A_{i},(i=1,2)$. Then the subgroup $H$ of $G$ generated by the retracts $H_{i}=A_{i} \phi_{i},(i=1,2)$, is the V-verbal product of $H_{1}$ and $\mathrm{H}_{2}$.

Now suppose $H_{1}$ and $H_{2}$ are retracts of $A_{1}$ and $A_{2}$, i.e., $H_{1}$ and $H_{2}$ have normal complements in $A_{1}$ and $A_{2}$ (in particular, suppose $A_{i}$ is a regular product $\left.A_{i}^{\prime} * H_{i} / N_{i}, i=(1,2)\right)$. Let $V$ be a verbal subgroup of $A_{1} * A_{2}$ and let $G=$ $A_{1} *_{V} A_{2}$ be the $V$-verbal product of $A_{1}$ and $A_{2}$. By the above Lemma $3.8 H=$ $H_{1} *_{V} H_{2}$, so $\tau$ exists by an argument similar to that given in (2). That is, by Theorem 2.4 an epimorphism $\tau: H \rightarrow H$ exists such that $\tau \mid H_{1}=\psi$ and $\tau \mid H_{2}=\psi^{-1}$. Finally, $\tau$ is an isomorphism because $\tau^{2}=1$.

Before continuing with further examples consider the following special case of Example (3) which shows that the amalgamated products will, in general, be different from each other as $V$ varies. (Of course, not always. Some amalgams can only generate their generalized free products; see Example 4.12 [4].)

Suppose $H_{1}$ and $H_{2}$ are $V$-verbal factors of $A_{1}$ and $A_{2}$, say $A_{1}=A_{1}^{\prime} *_{V} H_{1}$ and $A_{2}=A_{2}^{\prime} *_{V} H_{2}$. Let $G=A_{1} *_{V} A_{2}$. Then $H=H_{1} *_{V} H_{2}$ is a $V$-verbal factor of $G, G=\left(A_{1}^{\prime} *_{V} A_{2}^{\prime}\right) *_{V} H$, by the properties of $V$-verbal multiplication. Furthermore, the switching map $\tau$ as defined above can be extended to an automorphism $\tau^{\prime}$ of $G$ of order two such that $\tau^{\prime} \mid A_{1}^{\prime} *_{V} A_{2}^{\prime}$ is the identity on $A_{1}^{\prime} *_{V} A_{2}^{\prime}$ by Theorem 2.4. Choose $Z$ to be the normal complement $\left(A_{1}^{\prime} *_{V} A_{2}^{\prime}\right)^{G}$ of $H$ in $G$, and let $\sigma=\tau^{\prime} \mid Z$. Since $Z=\left(A_{1}^{\prime} *_{V} A_{2}^{\prime}\right)\left[A_{1}^{\prime} *_{V} A_{2}^{\prime}, G\right], \sigma$ is a permutation on $Z$. It must be verified that if $A_{1}$ meets $\left(z \tau^{\prime}\right) H$, then $\left(A_{2} \backslash H_{2}\right) \cap z H$ is empty. Suppose $z \tau^{\prime} h=a_{1} \in A_{1}$ for some $z \in Z$. Applying $\tau^{\prime}$ to both sides of this equation, $z\left(h \tau^{\prime}\right)=a_{1} \tau^{\prime} \in A_{1} *_{V} H_{2}$. Thus $z=a_{1}^{*} h_{2}^{*} c^{*}$ for some $a_{1}^{*} \in A_{1}, h_{2}^{*} \in H_{2}, c^{*} \in\left[A_{1}, A_{2}\right]$. If also $z h^{*}=a_{2} \in$ $A_{2} \backslash H_{2}$, then $z=h_{1}^{\prime} a_{2}^{\prime} c^{\prime}$, where $h_{1}^{\prime} \in H_{1}, a_{2}^{\prime} \in A_{2} \backslash H_{2}$ and $c^{\prime} \in\left[A_{1}, A_{2}\right]$. This would imply the contradiction $a_{2}^{\prime}=h_{2}^{*} \in H_{2}$. Thus $\left(A_{2} \backslash H_{2}\right) \cap z H$ is empty.

Now we show

$$
P=P\left(G, Z, \tau^{\prime} \mid Z\right) \cong A_{1}^{\prime} *_{V} H_{1} *_{V} A_{2}^{\prime} .
$$

Let $u \in A_{2}^{\prime}$ and $z h \in G$, where $z \in Z, h \in H$. Then

$$
\begin{aligned}
(z h) \rho_{u}^{\pi} & =\left((z h) \tau^{\prime} u\right) \pi & & \left(\sigma=\tau^{\prime} \mid Z\right) \\
& =\left((z h u) \tau^{\prime}\right) \tau^{\prime} & & \left(\tau^{\prime} \mid A_{2}^{\prime} \text { is the identity on } A_{2}^{\prime}\right) \\
& =(z h) \rho_{u}, & &
\end{aligned}
$$


that is, $P$ is generated by $A_{1} \rho$ and $\left(A_{2}^{\prime} \rho\right)^{\pi}=A_{2}^{\prime} \rho$. But these are just the right regular representations of $A_{1}$ and $A_{2}^{\prime}$ over $G$, which generate the right regular representation of $A_{1} *_{V} A_{2}^{\prime}$ over $G$, conpleting the proof of (3.9).

Note the condition that $H_{1}$ and $H_{2}$ be retracts in (3) is not necessary in order that $\tau$ exist; for example, Smel'kin [10] proved that if $A_{1}$ and $A_{2}$ are torsion free abelian groups and $V$ is the verbal subgroup of $A_{1} * A_{2}$ corresponding to the variety of nilpotent groups of class at most $n$, then $H=H_{1} *_{V} H_{2} \subseteq A_{1} *_{V} A_{2}$.

(4) Isomorphic Constituents. Suppose $A_{1}$ and $A_{2}$ are isomorphic, say $\gamma: A_{1} \cong A_{2}, \psi=\gamma \mid H_{1}$, and consider the $V$-verbal product $A_{1} *_{V} A_{2}$. Then $\tau$ exists, for there is an isomorphism $\tau^{\prime}$ of order two from $A_{1} *_{V} A_{2}$ onto $A_{1} *_{V} A_{2}$ such that $\tau^{\prime} \mid A_{1}=\gamma$ and $\tau^{\prime} \mid A_{2}=\gamma^{-1}$. Take $\tau=\tau^{\prime} \mid H$.

(5) Right Regular Representation. So far in the examples $A_{1} \cap A_{2}=$ $\{1\} \subseteq G$. At the other extreme, let $\mathscr{A}$ generate $G, H_{1}=H_{2}=H \subseteq G$, take $\tau$ as the identity on $H$; let $Z$ be any transversal of $H$ in $G$ containing 1 and let $\sigma$ be the identity on $Z$. Clearly $\pi$ is the identity and $P(G, H, i)$ is just the right regular representation of $G$. In particular, an amalgam $\mathscr{A}$ can generate a group $G$ if and only if $G$ is isomorphic to some amalgamated product on $\mathscr{A}$.

(3.11) The General CaSe. Suppose now that the amalgam has more than two constituents. Suppose that for each $i \in I, A_{i}$ is a group having a subgroup $H_{i}$ which is isomorphic to a fixed group $H^{\prime}$, say $\psi_{i}: H_{i} \cong H^{\prime}$ and set $\psi_{i j}=\psi_{i} \psi_{j}^{-1}: H_{i} \cong$ $H_{j}, i, j \in I, i \neq j$. Let $G$ be any group containing isomorphic copies $A_{i}^{*}$ of $A_{i}$, say $\phi_{i}: A_{i} \cong A_{i}^{*},(i \in I)$, and suppose $A_{i}^{*} \cap A_{j}^{*}=H_{i}^{*} \cap H_{j}^{*}$ and $\phi_{i}^{-1} \psi_{i j} \phi_{j}$ acts as the identity on $H_{i}^{*} \cap H_{j}^{*},(i, j \in I, i \neq j)$. Let $H$ be the subgroup of $G$ generated by the $H_{i}^{*},(i \in I)$, and assume $H \cap A_{j}^{*}=H_{j}^{*}, j \in \Lambda\{1\}$. Choose a transversal $Z$ of $H$ in $G$ and assume automorphisms $\tau_{j}$ can be defined on $H$ such that $\tau_{j} \mid H_{1}^{*}=\psi_{1 j}^{*}$, $\tau_{j} \mid H_{j}^{*}=\psi_{1 j}^{-1}$ and $\tau_{j} \mid H_{k}^{*}$ acts as the identity on $H_{k}^{*},(j, k \in I \backslash\{1\}, k \neq j)$. Define a permutation $\sigma$ on $Z$ as before, except assume for all $i, j \in I, i \neq j$, if $A_{j}^{*}$ meets $z H$, then both $\left(A_{i}^{*} \backslash H_{i}^{*}\right) \cap(z \sigma) H$ and $\left(A_{i}^{*} \backslash H_{i}^{*}\right) \cap z H$ are empty. Finally, for each $j \in I\{1\}$, let $\pi_{j}$ be a permutation on $G$ given by $(z h) \pi_{j}=(z \sigma)\left(h \tau_{j}\right), z \in Z, h \in H$; assume also that $\left(1 \pi_{j}\right) A_{j}=A_{j} H,(j \in I\{1\})$. Then, as before, the amalgam is isomorphic to $\cup\left\{\left(A_{i}^{*} \rho\right)^{\pi_{i}} \mid i \in I\right\}$, where $\pi_{1}$ is defined to be the identity on $G$. The details are omitted.

\section{An epimorphism}

Let $\mathscr{A}=A m\left(A_{i}, H_{i} ; \psi_{i j} ; i, j \in I\right)$ be an amalgam and let $G$ be a group containing copies $A_{i}^{*}$ of the $A_{i}$ as in Section (3.11).

Assume further that $G$ is generated by the $A_{i}^{*}$ and let $P=P(G, Z, \sigma)$ be an amalgamated product on $\mathscr{A}$. A homomorphism $\theta$ of $G$ will be called a $(G, Z, \sigma)$ homomorphism, if the following conditions are satisfied: 
(i') there exist isomorphisms $\psi_{i j}^{\prime}: H_{i} \theta \cong H_{j} \theta$ such that $\theta \psi_{i j}^{\prime}=\psi_{i j} \theta$, on $H_{i}$ $(i, j \in I, i \neq j)$.

(ii') $Z \theta$ is a transversal of $H \theta=\left\langle H_{i} \theta \mid i \in I\right\rangle$ in $G \theta$.

(iii') a permutation $\sigma^{\prime}: Z \theta \rightarrow Z \theta$ exists as required in order to construct a $\left(G \theta, Z \theta, \sigma^{\prime}\right)$-product on the factor amalgam $\mathscr{F}=A m\left(A_{i} \theta, H_{i} \theta, \psi_{i j}^{\prime} \mid i, j \in I\right.$, $i \neq j$ ), such that in addition $\theta \sigma^{\prime}=\sigma \theta$ on $Z$, and

(iv') for all $j \in I, j \neq 1$, switching maps $\tau_{j}^{\prime}: H \theta \rightarrow H \theta$ exist such that $\tau_{j}^{\prime} \mid H_{1} \theta=\psi_{1 j}^{\prime}$ and $\tau_{j}^{\prime} \mid H_{j} \theta=\left(\psi_{1 j}^{\prime}\right)^{-1}$.

Now suppose $\theta$ is such a $(G, Z, \sigma)$-homomorphism; then, since $H$ is generated by the $H_{i}, \theta \tau_{j}^{\prime}=\tau_{j} \theta$ on $H$. Furthermore, permutations $\pi_{j}^{\prime}: G \theta \rightarrow G \theta$ can be constructed as in (3.11) using $\sigma^{\prime}$ and $\tau_{j}^{\prime}$ and

$$
\theta \pi_{j}^{\prime}=\pi_{j} \theta, \quad(j \in I, j \neq 1) .
$$

Thus $\left(1 \pi_{j}^{\prime}\right) A_{j} \theta=\left(\left(1 \pi_{j}\right) A_{j}\right) \theta \subseteq\left(A_{j} H\right) \theta=A_{j} \theta H \theta$, which is required to construct a $\left(G \theta, Z \theta, \sigma^{\prime}\right)$-amalgamated product on $\mathscr{F}$ using the switching maps $\tau_{j}^{\prime}$. Denote the product depending on the $\psi_{i j}^{\prime}$ by $P^{\prime}\left(G \theta ; Z \theta, \sigma^{\prime}, \psi_{i j}^{\prime}\right)$ or merely by $P^{\prime}$.

THEOREM 4.2. Let $\mathscr{A}$ and $G$ be as above and suppose $\theta$ is a $(G, Z, \sigma)$-homomorphism of $G$. Then there exists an epimorphism $f$ from $P=P(G, Z, \sigma)$ onto $P^{\prime}=$ $P^{\prime}\left(G \theta, Z \theta, \sigma^{\prime}, \psi_{i j}^{\prime}\right)$ extending the canonical epimorphisms $\left(A_{i} \rho\right)^{\pi_{i}} \rightarrow\left(A_{i} \theta \rho\right)^{\pi^{\prime}},(i \in I)$.

Proof. The function $\theta$ is an epimorphism. It follows from (4.1) that for each $a_{j} \in A_{j}^{*}$

$$
\pi_{j} P_{a_{j}} \pi_{j} \theta=\theta \pi_{j}^{\prime} P_{a_{j} \theta} \pi_{j}^{\prime} \quad(j \in I)
$$

where, as in Section (3.11), $\pi_{1}$ and $\pi_{1}^{\prime}$ are the identities on $G$ and $G \theta$ respectively. Thus, since $P$ is generated by the $\left(A_{i}^{*} \rho\right)^{\pi_{i}}$, to each $x \in P$, there exists a unique $x f \in P^{\prime}$ such that $x \theta=\theta(x f) ; x f$ is unique because $\theta$ is an epimorphism. The required epimorphism $f$ is given by $f: x \rightarrow x f$. (cf. Theorem 3.1, [4]).

We shall call $f$ the natural homomorphism from $P$ onto $P^{\prime}$ when it exists.

The usual proof of the following well-known result uses directly the uniqueness of the normal form in the generalized free product.

COROLLARY 4.4. Let $G$ be any group gentrated by $\mathscr{A}$. Then there exists a natural homomorphism from the generalized free product on $\mathscr{A}$ onto $G$ which acts as the identity on the $A_{i},(i \in I)$.

Proof. (See Example (3.6), (2) and (5).) Consider the right regular representation of $G, G \rho$ as a product on $G$. There is a natural homomorphism $\theta$ from $F=\pi^{*}\left\{A_{i} \mid i \in I\right\}$ onto $G$ extending the maps $A_{i} \rightarrow A_{i} \subseteq G$. Let $Z=Z_{1} Z_{2}$ where $Z_{2}$ is a transversal of $H$ in $H \operatorname{ker} \theta$ such that $1 \in Z_{2} \subseteq \operatorname{ker} \theta$ and $Z_{1}$ is a transversal of $H \operatorname{ker} \theta$ in $F, 1 \in Z_{1}$. Then $Z$ is a transversal of $H$ in $F$ which maps onto a transversal $Z \theta$ of $H \theta$ in $F \theta=G$. Let $\sigma$ be the identity on $Z$. Then if $\sigma^{\prime}, \psi_{i j}^{\prime}$ and 
$\tau_{j}^{\prime}$ are taken to be identity maps, $\theta$ is a $(G, Z, \sigma)$-homomorphism, so the result follows by Theorem 4.2.

Note. Many times it will be convenient to choose $Z$ as above in Corollary 4.4; this will be denoted by a remark such as 'let $Z=Z_{1} Z_{2} \cdots$ ', if no further explanation is required. If no mention of $\sigma$ is made it will be assumed to be the identity on $Z$.

Now consider an amalgam on two groups $A_{1}$ and $A_{2}$. Let $G=A_{1} *_{V} A_{2}$ be a verbal product. Choose a transversal $Z_{1} Z_{2}$ of $H$ in $G$ as follows: let $Z_{2}$ be a transversal of $H$ in $H N, 1 \in Z_{2} \subseteq N$, where $N$ is the normal closure of the amalgamating relations $\left\{h_{1}^{-1}\left(h_{1} \psi\right) \mid h_{1} \in H_{1}\right\}$ in $G$ and let $Z_{1}$ be a transversal of $H N$ in $G, 1 \in Z_{1}$. (See Theorem 2.6.)

Corollary 4.5. Let $G=A_{1} *_{V} A_{2}$ and $Z_{1} Z_{2}$ be as above. If some $P=$ $P\left(G, Z_{1} Z_{2}, \sigma\right)$ exists which is a generalized $V$-verbal product on $\mathscr{A}$, then $P$ is the free generalized $V$-verbal product on $\mathscr{A}$.

Proof. Let $K$ be the free generalized $V$-verbal product on $\mathscr{A}$ and let $\theta: G \rightarrow K$ be the natural epimorphism from $G$ onto $K$. Then $Z \theta$ is a transversal of $H_{1}$ in $K$.

Thus there is a natural epimorphism $f$ from $P$ onto $K \rho$. If $\psi$ is the canonical epimorphism from $K \rho$ onto $P$, then $\psi f$ is the identity, so $P \cong K$ which was to be shown.

THEOREM 4.6. Let $G=A_{1} * A_{2} / N$ be any regular product. If any amalgamated product exists on $G$ which is generated by the amalgam $\mathscr{A}$, then a $(G, Z, i)$-amalgamated product exists which is a generalized regular product on $\mathscr{A}$.

Proof. Since at least one amalgamated product exists, the switching map exists. Let $Z$ be any transversal of $H$ in $G$ which maps onto a transversal $Z \theta=$ $S \times T$ of $H_{1} \times H_{2}$ in $A_{1} \times A_{2}$, where $\theta$ is the canonical epimorphism from $G$ onto $A_{1} \times A_{2}$. Then an amalgamated product $P=P(G, Z, i)$ exists and maps onto the permutational product $P^{\prime}=P(A \times B ; S \times T)$, say $\phi: P \rightarrow P^{\prime}$. Let $f$ and $f^{\prime}$ be the natural epimorphisms from the generalized free product on the amalgam onto $P$ and $P^{\prime}$, respectively. Since

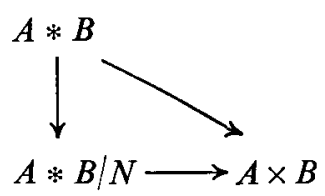

is a commutative diagram (where the maps are the canonical epimorphisms) it follows from Theorem 4.2 that $f^{\prime}=f \phi$, so $\operatorname{ker} f \subseteq \operatorname{ker} f^{\prime}$. Allenby [2] has shown that any permutational product is a generalized regular product, hence $P$ is itself a generalized regular product on the amalgam.

It is known that if the generalized direct product $D$ on $\mathscr{A}=\operatorname{Am}\left(A, B ; H_{1}\right.$, 
$\left.H_{2} ; \psi\right)$ exists, then all permutational products must be isomorphic to $D$, that is, $D$ is the free generalized abelian product on $\mathscr{A}$. The following examples show that even though the free generalized $V$-product generated by $\mathscr{A}$, say $K$, exists, and an amalgamated product $P=P\left(G, Z_{1} Z_{2}, i\right)$ exists on $A *_{V} B$ where the transversal $Z_{1} Z_{2}$ is chosen as in Corollary 4.5 (so $P$ is a generalized regular product mapping onto $K$ ), $P$ may not be isomorphic to $K$. (In this example $K$ will exist, because the generalized direct product does; see Wiegold [11], Theorem 4.6.)

Let $N_{c}$ stand for the verbal subgroup of $A * B$ corresponding to the class of nilpotent groups of class at most $c$.

Let $A \otimes B$ denote the tensor product of the groups $A$ and $B$. The regular $N_{2}$ product of groups $A$ and $B$ can be faithfully represented by

$$
G=\{(a, b, c) \mid a \in A, b \in B, c \in A \otimes B\},
$$

where

$$
(a, b, c)\left(a_{1}, b_{1}, c_{1}\right)=\left(a a_{1}, b b_{1}, c c_{1} a_{1}^{-1} \otimes b\right)
$$

and

$$
A \cong\{(a, 0,0) \mid a \in A\}, \quad B \cong\{(0, b, 0) \mid b \in B\}
$$

(Wiegold [11], p. 154).

EXAMPLE (4.7). If $A$ and $B$ are copies of the additive group of rational numbers, $Q$, then (using additive notation)

where

$$
G=\{(s, t, u) \mid s, t, u \in Q\}
$$

and

$$
(s, t, u)\left(s_{1}, t_{1}, u_{1}\right)=\left(s+s_{1}, t+t_{1}, u+u_{1}-t s_{1}\right),
$$

Let

$$
(s, t, u)^{-1}=(-s,-t,-u-t s) .
$$

$$
H_{1}=\{(2 n, 0,0) \mid n \in I\}, \quad H_{2}=\{(0,3 m, 0) \mid m \in I\},
$$

where $I$ is the integers, and assume the amalgamating isomorphism $\psi$ is given by $(2 n, 0,0)=$ ' $(0,3 n, 0), n \in I$. Now

$$
[(2,0,0),(0,3,0)]=(0,0,6)
$$

so

$$
\left\langle H_{1}, H_{2}\right\rangle=\{(2 n, 3 m, 6 p) \mid n, m, p \in I\} .
$$

The switching map $\tau$ exists by the remark at the end of (3.6) (3).

If $h_{1}=(2 \mathrm{n}, 0,0), n \in I$ then $h_{1}\left(h_{1}^{-1} \psi\right)=(2 n,-3 n, 0) \in N$, where $N$ is the normal closure of $\left\{h_{1}\left(h_{1}^{-1} \psi\right) \mid h_{1} \in H_{1}\right\}$ in $G$,

and

$$
(2 n,-3 n, 0)^{(s, t, u)}=(2 n,-3 n, 2 n t+3 n s) \in N
$$

where $s, t, u, \in Q$.

$$
(2,-3, u)(-2,3,0)=(0,0, u-6) \in N,
$$


Thus

and

$$
N=\{(2 n,-3 n, u) \mid n \in I, u \in Q\}
$$

$$
H N=\{(2 n, 3 m, u) \mid n, m \in I, u \in Q\} .
$$

If $u \in Q$, then $u$ can be uniquely written $u=6 k+u^{\prime}, 0 \leqq u^{\prime}<6, k \in I, u^{\prime} \in Q$. Choose the transversal $Z_{2}$ of $H$ in $H N$ to be

$$
Z_{2}=\left\{\left(0,0, u^{\prime}\right) \mid 0 \leqq u^{\prime}<6, u^{\prime} \in Q\right\} .
$$

Similarly choose a transversal $Z_{1}$, of $H N$ in $G$; let

$$
Z_{1}=\left\{\left(s^{\prime}, t^{\prime}, 0\right) \mid 0 \leqq s^{\prime}<2,0 \leqq t^{\prime}<3, s^{\prime}, t^{\prime} \in Q\right\} .
$$

Then

$$
Z_{1} Z_{2}=\left\{\left(s^{\prime}, t^{\prime}, u^{\prime}\right) \mid 0 \leqq s^{\prime}<2,0 \leqq \tau^{\prime}<3,0 \leqq u^{\prime}<6, s^{\prime}, \tau^{\prime}, u^{\prime} \in Q\right\}
$$

is a transversal of $H$ in $G$ chosen as required in Corollary 4.5.

If $z=(0,0,6 p)=(0,0,6)^{p} \in H$, then by $(4.8) z \tau=(0,0,6) \tau^{p}=[(0,3,0)$, $(2,0,0)]^{p}=(0,0,-6)^{p}$. Thus if $(2 m, 3 m, 6 p) \in H$,

$$
(2 n, 3 m, 6 p) \tau=(2 m, 3 n,-6 p-6 m n) .
$$

Since $\sigma=i$ on $Z_{1} Z_{2},(s, t, u) \pi$ can now be calculated for any $(s, t, u) \in G$.

Let $a^{\prime}=(1,0,0) \rho$ and $b^{\prime}=(0,1,0) \rho^{\pi}$. Then $\left(a^{\prime}\right)^{2} \in H \rho \subseteq Z(P)$; set $d$ equal to $a^{\prime}$ in Lemma 2.7 and $g=\left(b^{\prime}\right)^{\frac{1}{2} r}=\left(0, \frac{1 r}{2}, 0\right) \rho^{\pi}$. Then $\left[b^{\prime}, a^{\prime}\right] \in G_{(r+1)}, r \geqq 0$. Calculating,

$$
\left(\frac{1}{2}, \frac{5}{2}, 5\right)\left[b^{\prime}, a^{\prime}\right]=\left(\frac{1}{2}, \frac{5}{2}, 6\right) \text {. }
$$

Thus $P$ is not nilpotent of any class, so $P$ is not isomorphic to the free generalized nilpotent product of class $2, K$.

Suppose now the generalized $N_{2}$-product of an amalgam $\mathscr{A}$ exists. Does the existence of this product force the switching automorphism to exist in $A *_{N_{2}} B$ ?

The following example due to $\mathrm{Dr}$ L. G. Kovács shows this is not the case. Let $A=C_{2} \times C_{4}$ and $B=C_{2} \times C_{2}$, where $C_{n}$ is the cyclic group of order $n$; let these cyclic groups be generated by $a, b, c$ and $d$, respectively. Amalgamate $\left\langle a, b^{2}\right\rangle$ with $B$ via $a \leftrightarrow c, b^{2} \leftrightarrow d$. Then in $G=A *_{N_{2}} B$ we have $\left[b^{2}, e\right]=\left[b, e^{2}\right]$ $=1, e \in\{c, d\}$, so $b^{2}$ is in the centre of $G$ and thus of $H$. A simple calculation using Wiegold's representation of $G$ above shows that $d$ does not commute with $a$. Thus a switching automorphism does not exist. I thank Dr. Kovács for allowing me to use this example.

\section{A wreath product embedding}

It is convenient to generalize and unify the embeddings given in Theorems 4.1, 5.2 and 6.1 of $[4]$ in the following way.

Assume that an amalgam $\mathscr{A}$ is given as in (3.11) and that some amalgamated 
product $P=P(G, Z, \sigma)$ on a group $G$ exists generated by $\mathscr{A}$. Let $\theta$ be a $(G, Z, \sigma)$ homomorphism, $P^{\prime}=P^{\prime}\left(G \theta, Z \theta, \sigma^{\prime}\right)$ and $f: P \rightarrow P^{\prime}$ the natural homomorphism. Choose a set $W$ of coset representatives of $\operatorname{ker} \theta$ in $G$. Thus, if $d \in G$, then $d=w \lambda$, $w \in W, \lambda \in \operatorname{ker} \theta$ and $d \theta=w \theta$. Define $[d \theta]=w$ and note $[d \theta] \theta=d \theta$.

TheOrem 5.1. Suppose there exist homomorphisms $\alpha: P \rightarrow \operatorname{Aut}(\operatorname{ker} \theta)$ and $r: \operatorname{ker} \theta \rightarrow \mathscr{S}(G)$ such that

(1) if $g \in G$, then there exists a unique $\lambda r \in(\operatorname{ker} \theta) r=R$ such that $g=[g \theta] \lambda r$, (2) if $y \in P$, then

$$
y^{-1}(\lambda r) y=\left(\lambda^{y \alpha}\right) r
$$

and $(\operatorname{ker} r)^{y x} \subseteq \operatorname{ker} r(y \in P)$.

Then there exists a monomorphism from $P$ into the unrestricted permutational wreath product

$$
P \beta(\operatorname{ker} \theta) r W r\left(P^{\prime} ; G \theta\right) \text {. }
$$

where the homomorphism $\beta: P \rightarrow \operatorname{Aut}((\operatorname{ker} \theta) r)$ is given by

$$
\lambda r^{y \beta}=\left(\lambda^{y \alpha}\right) r \quad(\lambda \in \operatorname{ker} \theta, y \in P) .
$$

Proof. First note that (5.4) determines a homomorphism $\beta$ as required.

Now let $x \in P$. It follows from the proof of Theorem 4.2 if $d \in G$, then

$$
\mathrm{d} x \theta=d \theta x f,
$$

so $[d \theta] x \theta=d \theta x f$. Thus, by (1), if $d \in G$, there exists a unique $\left(\lambda_{x}(d \theta)\right) r \in(\operatorname{ker} \theta) r$ such that

$$
[d \theta] x=[d \theta x f]\left(\lambda_{x}(d \theta)\right) r .
$$

Define an element $e_{x}$ in the direct power of $|G \theta|$ copies of $P \beta(\operatorname{ker} \theta) r$, $(P \beta(\operatorname{ker} \theta) r)^{G \theta}$, by

$$
e_{x}(d \theta)=x \beta\left(\lambda_{x}^{-1}(d \theta)\right) r . \quad(d \theta \in G \theta)
$$

LEMMA 5.8. The required monomorphism is given by

$$
x \rightarrow x f e_{x}^{x f}=e_{x} x f \quad(x \in P) .
$$

Proof. It must be shown that

or

$$
\begin{aligned}
e_{x y}(x y) f & =e_{x} x f e_{y} y f \\
e_{x y} & =e_{x} e_{y}^{x f^{-1}}
\end{aligned}
$$

which by the definition of conjugation in wreath products is equivalent to

$$
e_{x y}(d \theta)=e_{x}(d \theta) e_{y}(d \theta x f) \quad(d \theta \in G \theta) .
$$

Now by (1) and repreated use of (5.6), if $d=[d \theta] \lambda r \in G$, with $\lambda \in \operatorname{ker} \theta$, then 


$$
\begin{aligned}
([d \theta] \lambda r) x y & =[d \theta x f y f]\left(\Lambda_{1}\right) r \\
& =[d \theta x f] y\left(\Lambda_{2}\right) r \\
& =[d \theta] x\left(\lambda_{x}^{-1}(d \theta)\right) r y\left(\Lambda_{2}\right) r \\
& =[d \theta] x y\left(\Lambda_{3}\right) r \\
& =([d \theta] \lambda r)\left(\lambda^{-1}\right) r x y\left(\Lambda_{3}\right) r \\
& =([d \theta] \lambda r) x y\left(\Lambda_{4}\right) r,
\end{aligned}
$$

where

$$
\begin{aligned}
& \Lambda_{1}=\lambda_{x y}(d \theta) \lambda^{(x y) \alpha} \\
& \Lambda_{2}=\lambda_{y}^{-1}(d \theta x f) \Lambda_{1} \\
& A_{3}=\lambda_{x}^{-y \alpha}(d \theta) \Lambda_{2}
\end{aligned}
$$

and

$$
\Lambda_{4}=\lambda^{-(x y) \alpha} \Lambda_{3}=\lambda^{-(x y) \alpha} \lambda_{x}^{-y \alpha}(d \theta) \lambda_{y}^{-1}(d \theta x f) \lambda_{x y}(d \theta) \lambda^{(x y) \alpha} .
$$

Thus $\left(\Lambda_{4}\right) r=1$, so

$$
\left(\lambda_{x}^{-y \alpha}(d \theta)\right) r\left(\hat{\lambda}_{y}^{-1}(d \theta x f)\right) r\left(\lambda_{x y}(d \theta)\right) r=1
$$

from which (5.10) follows.

To complete the proof suppose $e_{x} x f=1$. Then $x f=1, x \beta=1$ and for each $d \theta \in G \theta,\left(\lambda_{x y}(d \theta)\right) r=1$. Let $d=[d \theta] \lambda r \in G$. Then

$$
\begin{aligned}
([d \theta] \lambda r) x & =[d \theta] x \lambda r^{x \beta} \\
& =[d \theta x f]\left(\lambda_{x}(d \theta)\right) r \lambda r^{x \beta} \\
& =[d \theta] \lambda r .
\end{aligned}
$$

Thus $x=1$ completing the proof.

For example, if Theorem 5.1 is applied to permutational products where $H_{1} \triangleleft A$ and $H_{2} \triangleleft B$, then $\operatorname{ker} \theta=H_{1} \times H_{2}, r$ can be chosen to be the restriction of the right regular representation of $G$ to $H_{1} \times H_{2}$ and if $y=\rho_{a_{1}} \rho_{b_{2}}^{\pi} \cdots \rho_{a_{n}} \in P$, where $a_{i} \in A, b_{j} \in B$, then $\alpha$ is given by the equations $y^{-1}\left(\rho_{h_{1} h_{2}}\right) y=\rho_{u}$, where $u=h_{1}^{z} h_{2}, h_{1} h_{2} \in H_{1} \times H_{2}$, and (with the obvious meaning), $z=a_{1} b_{2} \cdots a_{n}$. This is essentially the embedding Theorem 4.1 of [4] mentioned at the beginning of this section. It can be shown that, in general, the term $P \beta$ is needed for permutational products. On the other hand, the following shows why $r$ is not always set equal to $\rho$ as above.

If $G^{*}$ is the generalized free product of $A$ and $B$ above ( $H$ normal in each), then there is a homomorphism $\theta: G^{*} \rightarrow A / H * B / H$ such that ker $\theta=H$. Considering both the right regular representations of $G^{*}$ and $A / H * B / H$ as amalgamated products on $G^{*}$ and $A / H * B / H$, and taking $r=\rho$ as above, $G^{*}$ can be embedded in $P \beta H W r A / H * B / H$, where $P \beta$ is the group of automorphisms $G^{*}$ induces on $H, G^{*} / C_{G^{*}}(H)$. This is not as good as the standard wreath product embedding of $G^{*}, H W r A / H * B / H$. Instead if $g^{*} \in G^{*}$, define $\left(g^{*}\right) \lambda r=\lambda^{-1} g^{*}$. Then $H r$ commutes with $P$ in $\mathscr{S}(G)$. This choice of $r$ in (5.1) thus gives the expected embedding of $G^{*}$. 
It is also not difficult to see that Theorem 6.10 of [4] can also be extended to amalgamated products. That is, suppose $P(G, Z, \sigma)$ is an amalgamated product on $\mathscr{A}, H_{1} \subseteq U_{1} \subseteq A_{1}, H_{2} \subseteq U_{2} \subseteq A_{2}$, and assume $Z$ is chosen as in [4], i.e., $Z=Z_{1} Z_{2}$, where $Z_{1}$ is a transversal of $U$ in $G$, where $U=\left\langle U_{1}, U_{2}\right\rangle$, and $Z_{2}$ is a transversal of $H$ in $U, 1 \in Z_{1} \cap Z_{2}$.

Then, if $\sigma$ sends $z_{1} z_{2}$ to $z_{1} z_{2}^{\prime}, z_{1} \in Z_{1}, z_{2}, z_{2}^{\prime} \in Z_{2}$, the subgroup $U^{*}$ of $P$ generated by $U_{1} \rho$ and $\left(U_{2} \rho\right)^{\pi}$ is isomorphic to $P_{1}\left(U, Z_{2}, \sigma \mid Z_{2}\right)$.

We conclude by stating two of the many problems which suggest themselves here and which we have not been able to answer.

(1) It is known that not every subgroup $U^{*}$ of a permutational product (i.e., an amalgamated product on $A \times B$ ) need again be a permutational product even though it is generated by $U_{1} \subseteq A_{1}$ and $U_{2} \subseteq A_{2}$, where $U_{1} \cap H_{1}=U_{2} \cap H_{1}$ ([9]). Suppose $U_{1}$ and $U_{2}$ are so chosen in an amalgamated product $P$ on a regular product $A * B / N$, such that (i) $P$ is a generalized regular product and (ii) the subgroup $U^{*}$ of $P$ is a generalized regular product (Allenby [1] gives some general criteria for this to happen). When must the subgroup $U^{*}$ be an amalgamated product on a regular product $U_{1} * U_{2} / N_{1}$ (where $N_{1}$ is possibly different from $N$ )?

(2) Determine some classes of amalgamated products on verbal products $A *_{V} B$ which are generalized $V$-verbal products, other than those on $A * B$ and $A \times B$.

\section{References}

[1] R. B. J. T. Allenby, 'Normal forms for generalised regular products of groups', Math. Zeitschr. 102 (1967) 356-369.

[2] R. B. J. T. Allenby, 'Permutational Products and Regular Products of Groups', Trans. Amer. Math. Soc. 13 (1969) 537-543.

[3] O. N. Golovin, 'Nilpotent products of groups', Mat. Sb. 27 (69) (1950), 427-454-Amer. Math. Soc. Transl. Ser. II 2 (1956), 89-115.

[4] R. J. Gregorac, 'On Permutational Products of Groups', J. Austral. Math. Soc., 10 (1969), $111-135$.

[5] S. Moran, 'Associative operations on groups, I', Proc. London Math. Soc. (3) 6 (1956), $581-596$.

[6] S. Moran, 'Associative operations on groups, II', Proc. London Math. Soc. (3) 8 (1958), $548-568$.

[7] B. H. Neumann, 'An essay on free products of groups with amalgamations' Phil. Trans. Royal Soc. of London (A) 246 (1954), 503-554.

[8] B. H. Neumann, 'Permutational Products of Groups', J. Austral. Math. Soc. 1, (1960) 299310.

[9] B. H. Neumann, 'On Amalgams of Periodic Groups', Proc. Roy. Soc. (A) 255 (1960), $477-489$.

[10] A. L. Šmel'kin, 'Nilpotent products and torsion-free nilpotent groups', Sibirsk. Math. Journal, 3, No. 4 (1962) 625-640.

[11] J. Wiegold, 'Nilpotent products of groups with amalgamations', Publ. Math. Debrecen 6 (1959), 131-168.

[12] J. Wiegold, 'Some remarks on generalised products of groups with amalgamations', Math. Zeitschr. 75 (1961), 57-78.

Institute of Advanced Studies

Australian National University, Canberra 